\title{
Epidemiología de trastornos mentales infanto-juveniles en la Provincia de Cautín
}

\author{
BENJAMÍN VICENTE ${ }^{1}$, SANDRA SALDIVIA ${ }^{\text {la }}$, PEDRO RIOSECO ${ }^{1}$, \\ FLORA DE LA BARRA ${ }^{2}$, MARIO VALDIVIA ${ }^{1}$, ROBERTO MELIPILLAN ${ }^{1 b}$, \\ MARIO ZÚÑIGA ${ }^{1}$, BERTHA ESCOBAR ${ }^{3 c}$, ROLANDO PIHAN ${ }^{1}$
}

\section{Prevalence of mental health disorders among children and adolescents living in southern Chile}

Background: The knowledge about prevalence of psychiatric disorders in all age groups is fundamental to plan an adequate mental health care. Aim: To determine the prevalence of DSM-IV psychiatric disorders in a representative sample of children and adolescents living in the province of Cautin, Chile. Material and Methods: Subjects aged between 4 and 18 years were selected in an aleatory stratified multistage fashion. As part of a national sample, four counties in Cautin were selected, then blocks, homes and the child or adolescent to be interviewed. Psychology graduate students applied the Spanish computer version of DISC-IV, as well as a questionnaire on family risk factors, socioeconomic index and service use. Results: A sample of 272 children and adolescents was evaluated, obtaining 93,4\% of participation. Using the most stringent impairment algorithm, the prevalence rate for any psychiatric disorder was $16.8 \%$, being $16.5 \%$ in boys and $17.1 \%$ in girls and mainly explained by anxiety and affective disorders. Prevalence was practically the same in the group aged 4 to 11 years than in those aged 12 to 18 years (16.7\% and $16.8 \%$ respectively). Prevalence of anxiety disorders was the highest, but less associated with impairment. On the other hand, all children and adolescents with affective disorders were impaired. Conclusions: The prevalence of psychiatric disorders in Cautin is high in children and adolescents.

(Rev Med Chile 2010; 138: 965-973).

Key words: Adolescent; Child, preschool; Mental health; Mental disorders.

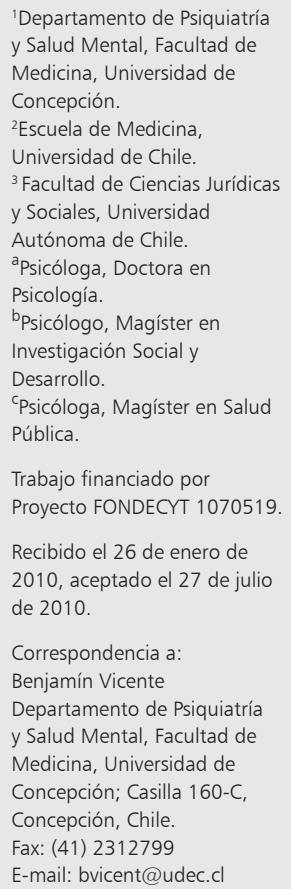

$\mathrm{E}$ 1 conocimiento de la prevalencia de los trastornos psiquiátricos en la población general resulta fundamental para la planificación racional de los recursos para atender dicha demanda. En Chile se cuenta con estudios epidemiológicos en población adulta de gran calidad metodológica, uno de ellos representativo de la población nacional $^{1-3}$. Sin embargo, no hay estudios equivalentes en la población de niños y adolescentes.

De esta forma, toda la planificación en Salud Mental Infanto-Juvenil se basa en información a partir de dos fuentes. Una, estudios internacionales como los de Anderson y cols en Nueva Zelandia ${ }^{4}$, Bird y cols en Puerto Rico ${ }^{5}$, McGee y cols ${ }^{6}$, Puura y cols en Finlandia ${ }^{7}$ y Canino y cols en Puerto Rico $^{8}$, que muestran prevalencia de trastornos psiquiátricos en la población infanto- juvenil entre el 17 y $20 \%$.

La segunda fuente son estudios nacionales efectuados en muestras pequeñas y no representativas de la realidad nacional. Entre éstos está el estudio sobre una reducida muestra de alumnos que cursaban primero básico en establecimientos de tres comunas del área occidente de Santiago, a quienes se aplicó una entrevista psiquiátrica semi-estructurada ${ }^{9}$. Otros estudios realizados con muestras más amplias son sólo representativos de determinadas localidades ${ }^{10}$. Y, en general, 
todos han utilizado instrumentos que no arrojan diagnósticos psiquiátricos categoriales sino que sólo identifican problemas conductuales y emocionales generales o perfiles de riesgo, tales como el CBCL o el TOCA-R ${ }^{11,12}$. También se cuenta con estudios en población consultante en centros de especialidad ${ }^{13,14}$.

Ante esta ausencia de información epidemiológica fiable, el equipo de investigación epidemiológica del Departamento de Psiquiatría y Salud Mental de la Universidad de Concepción, con financiamiento FONDECYT, ha llevado a cabo el primer estudio de prevalencia de trastornos psiquiátricos en la población chilena entre $4 \mathrm{y}$ 18 años. Éste se diseñó sobre una muestra representativa de 4 provincias. Los resultados que se presentan corresponden a la provincia de Cautín. Los objetivos de esta publicación son conocer la prevalencia de trastornos psiquiátricos en una muestra representativa de la población entre 4 y 18 años de la provincia de Cautín, su distribución según género y grupo etáreo, y determinar la asociación de variables sociodemográficas con la presencia de los distintos trastornos psiquiátricos en la población estudiada.

\section{Método}

Se diseñó una muestra aleatoria probabilística que consideró una prevalencia de trastornos mentales en la población general entre 4 y 18 años de $18 \%$, considerando los hallazgos de Canino y col${ }^{8}$ para el estudio comunitario de prevalencias en población infanto-juvenil en Puerto Rico. Con una seguridad de $95 \%$ y un error máximo de 1,75, ésta alcanza a 272 sujetos en la provincia de Cautín.

La selección de los participantes se realizó con un muestreo probabilístico por conglomerados en cuatro etapas. El proceso de selección de la muestra consideró la subdivisión de las provincias en comunas y éstas en manzanas. Cada unidad se seleccionó al azar. Seleccionada la manzana, se contabilizó el número de hogares disponibles en cada una, excluyendo aquellos institucionales. Utilizando el censo nacional de 2002 se determinó el número de hogares requerido en cada manzana. Los hogares se eligieron en sentido horario, comenzando por el primero en la esquina norte de cada bloque o manzana. El orden de visita fue definido por muestreo aleatorio simple. El niño o adolescente con el cumpleaños más cercano a la fecha de la entrevista fue seleccionado. De existir dos con igual fecha se usó una moneda, en el evento que fueran tres las tablas Kish ajustadas por sexo y edad pre-asignadas al azar ${ }^{15}$.

Obtenidas las probabilidades de selección de las distintas etapas de muestreo se calculó el peso para cada entrevistado. Usando los datos del Censo 2002 se construyó un factor de ajuste por población, empleando la distribución poblacional en términos de género y edad. Este factor se utilizó para ajustar los pesos obtenidos inicialmente, de modo de aumentar la precisión de las estimaciones.

Los desórdenes psiquiátricos DSM-IV fueron evaluados usando el DISC-IV, que corresponde a la versión más reciente del Diagnostic Inteview for Children ${ }^{16}$ instrumento que está ampliamente validado en sus distintas versiones ${ }^{17-25}$ incluyendo aquellas en español ${ }^{8,26-28}$. La entrevista permite además determinar el nivel de discapacidad asociado, la que puede clasificarse en cuatro criterios que van desde $\mathrm{A}$ hasta $\mathrm{D}$, siendo inclusivos y de menor a mayor exigencia. En este artículo se presentan los resultados de acuerdo a diagnóstico DSM-IV, y éste unido al criterio de discapacidad $\mathrm{D}$, definido como la presencia de discapacidad en un ámbito con intensidad grave, de acuerdo al reporte del entrevistado, o la interferencia en dos ámbitos con una valoración de intensidad intermedia.

Las entrevistas se realizaron en el hogar, por entrevistadores previamente capacitados, aplicándose el instrumento directamente al adolescente, en el caso de los mayores de 12 años, $o$ a sus padres en los de 12 años o menos.

El análisis estadístico se realizó con las funciones para diseño de muestras complejas de STATA 11.0. Los datos fueron ingresados con doble digitación, buscando minimizar errores. Para calcular errores estándar de los estimadores de los parámetros se usó el método de linearización de Taylor. Además de las prevalencias se realizó análisis de regresión logística binaria para estudiar la asociación entre la presencia de diagnóstico y eventuales variables predictoras.

El estudio contó con la aprobación del comité de ética de la Universidad de Concepción y de FONDECYT. Se solicitó consentimiento informado de uno de los padres o adulto responsable; $y$, para entrevistados entre 12 y 18 años, del mismo entrevistado. 


\section{Resultados}

La tasa de respuesta alcanzó a 93,4\%, completándose satisfactoriamente 254 entrevistas. Si bien la muestra presenta una ligera diferencia en relación a la población en la distribución por genero y edad, éstas no son significativas ( $\mathrm{p}=0,297$ para edad; $\mathrm{p}=0,324$ para género) y se corrigen al pesar la muestra (Tabla 1).

La prevalencia general de los trastornos psiquiátricos estudiados, en los 12 meses previos al estudio, alcanzó a $28,1 \%$, cifra que disminuye a
$16,8 \%$ al aplicar el criterio para discapacidad.

Los trastornos ansiosos constituyen el grupo diagnóstico más frecuentes (18,9\%), disminuyendo a $8,7 \%$ al corregir por criterio de discapacidad. Al estudiar patologías especificas es posible observar prevalencias más altas de trastornos por ansiedad de separación $(9,5 \%)$ y trastorno de ansiedad generalizada (9,2\%); sin embargo, al corregir por criterio de discapacidad éstas bajan a $4,2 \%$ y $2,3 \%$, respectivamente, y el déficit atencional pasa a ser el más prevalente con $4,3 \%$ (Tabla 2).

Tabla 1. Características de la muestra en Cautín

\begin{tabular}{|c|c|c|c|}
\hline Cautín & Muestra sin pesar & Muestra pesada & Población \\
\hline Total (4 - 18 años) & 254 & & $189.134(100,0 \%)$ \\
\hline 4 - 11 Años & $136(53,5 \%)$ & $52,9 \%$ & $100.078 \quad(52,9 \%)$ \\
\hline 12 - 18 Años & $118(46,5 \%)$ & $47,1 \%$ & $89.056 \quad(47,1 \%)$ \\
\hline Masculino & $128(50,4 \%)$ & $50,9 \%$ & $96.243 \quad(50,9 \%)$ \\
\hline Femenino & $126(49,6 \%)$ & $49,1 \%$ & $92.891 \quad(49,1 \%)$ \\
\hline
\end{tabular}

Tabla 2. Prevalencia de diagnóstico psiquiátrico DSM-IV en el último año en la provincia de Cautín

\begin{tabular}{|c|c|c|c|c|}
\hline \multirow[t]{2}{*}{ Trastornos } & \multicolumn{2}{|c|}{$\begin{array}{c}\text { Diagnóstico según } \\
\text { DISC-IV }\end{array}$} & \multicolumn{2}{|c|}{$\begin{array}{c}\text { Diagnóstico + Criterio } \\
\text { para discapacidad }\end{array}$} \\
\hline & $\%$ & EE & $\%$ & EE \\
\hline Trastornos ansiosos & 18,9 & 3,0 & 8,7 & 2,4 \\
\hline Fobia social & 3,1 & 1,3 & 2,2 & 1,2 \\
\hline Trastorno de ansiedad generalizada & 9,2 & 2,7 & 2,3 & 1,6 \\
\hline Trastorno por ansiedad de separación & 9,5 & 1,2 & 4,2 & 1,9 \\
\hline Trastornos afectivos & 4,1 & 1,7 & 4,1 & 1,7 \\
\hline Trastorno depresivo mayor & 4,1 & 1,7 & 4,1 & 1,7 \\
\hline Distimia & - & - & - & - \\
\hline Trastornos por comportamiento disruptivo & 8,6 & 2,7 & 5,6 & 1,9 \\
\hline Trastorno disocial & 0,7 & 0,5 & 0,6 & 0,5 \\
\hline Trastornos oposicionista desafiante & 3,9 & 1,6 & 1,4 & 0,7 \\
\hline TDAH & 5,0 & 1,3 & 4,3 & 1,6 \\
\hline Trastornos por consumo de alcohol y sustancias & 3,6 & 1,3 & 1,2 & 0,8 \\
\hline Abuso de alcohol & 1,8 & 1,1 & 0,3 & 0,3 \\
\hline Dependencia de alcohol & 1,0 & 0,8 & 0,8 & 0,7 \\
\hline Abuso de THC & 0,2 & 0,2 & - & - \\
\hline Dependencia de THC & 0,2 & 0,2 & 0,2 & 0,2 \\
\hline Dependencia de nicotina & 1,2 & 0,9 & 0,2 & 0,2 \\
\hline Abuso de otras sustancias & 0,2 & 0,2 & - & - \\
\hline Dependencia de otras sustancias & 0,2 & 0,2 & - & - \\
\hline Esquizofrenia & 0,9 & 0,8 & - & \\
\hline Cualquier diagnóstico & 28,1 & 2,0 & 16,8 & 2,3 \\
\hline
\end{tabular}


La prevalencia global de patología psiquiátrica en varones alcanza a $28,7 \%$, que disminuye a $16,5 \%$ al utilizar el criterio de discapacidad. El 27,5\% de mujeres había presentado un diagnóstico psiquiátrico en el último año, ajustándose a un $17,1 \%$, usando criterio de discapacidad. Los trastornos ansiosos son los más frecuentes en ambos géneros, con $15,0 \%$ en varones y $23,1 \%$ en mujeres; sin embargo, al aplicar el criterio de discapacidad en mujeres continúa siendo el diagnóstico más frecuente $(11,2 \%)$, pero en varones los trastornos por comportamiento disruptivo pasan a ser más prevalentes $(6,8 \%)$.
Si se analizan las patologías específicas se observa que en mujeres son más prevalentes los trastornos por ansiedad generalizada $(13,1 \%)$ y por ansiedad de separación (10,3\%), pero al corregir según criterio de discapacidad aparece el trastorno depresivo mayor como más frecuente $(4,9 \%)$. En varones son más prevalentes los trastornos por ansiedad de separación $(8,6 \%)$ y el trastorno por déficit atencional $(6,7 \%)$. Sin embargo, esto se invierte al corregir por criterio de discapacidad (Tabla 3).

Al distribuir la población por grupos etarios la prevalencia global de cuadros psiquiátricos es

Tabla 3. Prevalencia de trastornos psiquiátricos DSM-IV en el último año en la provincia de Cautín, según género

\begin{tabular}{|c|c|c|c|c|c|c|c|c|}
\hline \multirow[t]{3}{*}{ Trastornos } & \multicolumn{4}{|c|}{ Varones } & \multicolumn{4}{|c|}{ Mujeres } \\
\hline & \multicolumn{2}{|c|}{$\begin{array}{l}\text { Diagnóstico } \\
\text { según } \\
\text { DISC-IV }\end{array}$} & \multicolumn{2}{|c|}{$\begin{array}{c}\text { Diagnóstico } \\
+ \text { Criterio } \\
\text { para } \\
\text { discapacidad }\end{array}$} & \multicolumn{2}{|c|}{$\begin{array}{l}\text { Diagnóstico } \\
\text { según } \\
\text { DISC-IV }\end{array}$} & \multicolumn{2}{|c|}{$\begin{array}{c}\text { Diagnóstico } \\
+ \text { Criterio } \\
\text { para } \\
\text { discapacidad }\end{array}$} \\
\hline & $\%$ & EE & $\%$ & EE & $\%$ & EE & $\%$ & EE \\
\hline Trastornos ansiosos & 15,0 & 2,0 & 6,3 & 1,2 & 23,1 & 5,1 & 11,2 & 3,7 \\
\hline Fobia social & 2,4 & 1,5 & 2,1 & 1,5 & 3,9 & 2,3 & 2,3 & 1,9 \\
\hline Trastorno de ansiedad generalizada & 5,5 & 1,2 & - & - & 13,1 & 4,4 & 4,8 & 3,1 \\
\hline Trastorno por ansiedad de separación & 8,6 & 2,1 & 4,2 & 2,4 & 10,3 & 1,7 & 4,2 & 1,8 \\
\hline Trastornos afectivos & 3,4 & 2,0 & 3,4 & 2,0 & 4,9 & 2,2 & 4,9 & 2,2 \\
\hline Trastorno depresivo mayor & 3,4 & 2,0 & 3,4 & 2,0 & 4,9 & 2,2 & 4,9 & 2,2 \\
\hline Distimia & - & - & - & - & - & - & - & - \\
\hline $\begin{array}{l}\text { Trastornos por comportamiento } \\
\text { disruptivo }\end{array}$ & 11,0 & 3,5 & 6,8 & 2,1 & 6,1 & 3,1 & 4,4 & 2,9 \\
\hline Trastorno disocial & 1,1 & 1,0 & 1,1 & 1,0 & 0,3 & 0,3 & - & - \\
\hline Trastornos oposicionista desafiante & 4,9 & 2,2 & 1,7 & 0,8 & 2,9 & 1,1 & 1,2 & 0,6 \\
\hline TDAH & 6,7 & 2,5 & 5,4 & 1,7 & 3,2 & 2,9 & 3,2 & 2,9 \\
\hline $\begin{array}{l}\text { Trastornos por consumo de alcohol } \\
\text { y sustancias }\end{array}$ & 6,2 & 2,0 & 1,6 & 1,5 & 1,0 & 0,3 & 0,7 & 0,6 \\
\hline Abuso de alcohol & 2,5 & 1,8 & - & - & 1,0 & 0,4 & 0,7 & 0,6 \\
\hline Dependencia de alcohol & 2,0 & 1,6 & 1,6 & 1,5 & - & - & - & - \\
\hline Abuso de THC & 0,3 & 0,4 & - & - & - & - & - & - \\
\hline Dependencia de THC & 0,4 & 0,5 & 0,4 & 0,5 & - & - & - & - \\
\hline Dependencia de nicotina & 2,4 & 1,6 & 0,4 & 0,5 & - & - & - & - \\
\hline Abuso de otras sustancias & 0,4 & 0,5 & - & - & - & - & - & - \\
\hline Dependencia de otras sustancias & 0,4 & 0,5 & - & - & - & - & - & - \\
\hline Esquizofrenia & 1,8 & 1,6 & - & - & - & - & - & - \\
\hline Cualquier diagnóstico & 28,7 & 3,5 & 16,5 & 2,6 & 27,5 & 4,3 & 17,1 & 2,8 \\
\hline
\end{tabular}


muy semejante entre niños de 4 a 11 años, 27,6\% que se ajusta a $16,7 \%$ al aplicar criterios de discapacidad, y adolescentes de 12 a 18 años, $28,7 \%$ que se ajusta a $16,8 \%$. Los trastornos ansiosos son el grupo diagnóstico más frecuente en ambos rangos etarios (19,0\% en niños y 18,9\% en adolescentes), pero al ajustar por criterios de discapacidad los trastornos por comportamiento disruptivos pasan a ser el diagnóstico más frecuente en el grupo de menor edad $(8,6 \%)$.

Si se analizan patologías específicas, el diagnóstico más frecuentes en niños de 4 a 11 años es el trastorno por déficit atencional, con $8,7 \%$ que se ajusta a 7,4\% al aplicar discapacidad; mientras que en los adolescentes es el trastorno de ansiedad generalizada, con $12,5 \%$ que disminuye hasta $2,9 \%$ al corregir por discapacidad, pasando a ser el trastorno por ansiedad de separación la patología más prevalente en los adolescentes $(8,0 \%)$ (Tabla 4$)$.

Los trastornos del ánimo tienen una prevalencia más baja que los trastornos ansiosos y son más frecuentes en el grupo adolescente. Todos los casos detectados con trastorno depresivo mayor, están asociados a discapacidad.

Los trastornos por abuso de sustancias se observan sólo en el grupo adolescente, con una

Tabla 4. Prevalencia de trastornos psiquiátricos DSM-IV en el último año en la provincia de Cautín, según grupo etario

\begin{tabular}{|c|c|c|c|c|c|c|c|c|}
\hline \multirow[t]{3}{*}{ Trastornos } & \multicolumn{4}{|c|}{ 4-11 años } & \multicolumn{4}{|c|}{ 12-18 años } \\
\hline & \multicolumn{2}{|c|}{$\begin{array}{l}\text { Diagnóstico } \\
\text { según } \\
\text { DISC-IV }\end{array}$} & \multicolumn{2}{|c|}{$\begin{array}{c}\text { Diagnóstico } \\
+ \text { Criterio } \\
\text { para } \\
\text { discapacidad }\end{array}$} & \multicolumn{2}{|c|}{$\begin{array}{l}\text { Diagnóstico } \\
\text { según } \\
\text { DISC-IV }\end{array}$} & \multicolumn{2}{|c|}{$\begin{array}{c}\text { Diagnóstico } \\
+ \text { Criterio } \\
\text { para } \\
\text { discapacidad }\end{array}$} \\
\hline & $\%$ & EE & $\%$ & EE & $\%$ & EE & $\%$ & EE \\
\hline Trastornos ansiosos & 19,0 & 3,9 & 6,7 & 1,6 & 18,9 & 9,1 & 10,9 & 5,2 \\
\hline Fobia social & 5,2 & 2,1 & 4,1 & 2,2 & 0,8 & 0,9 & - & - \\
\hline Trastorno de ansiedad generalizada & 6,4 & 2,3 & 1,8 & 1,4 & 12,5 & 6,7 & 2,9 & 2,9 \\
\hline Trastorno por ansiedad de separación & 9,6 & 2,4 & 0,8 & 0,9 & 9,3 & 3,9 & 8,0 & 3,4 \\
\hline Trastornos afectivos & 2,6 & 1,7 & 2,6 & 1,7 & 5,9 & 2,5 & 5,9 & 2,5 \\
\hline Trastorno depresivo mayor & 2,6 & 1,7 & 2,6 & 1,7 & 5,9 & 2,5 & 5,9 & 2,5 \\
\hline Distimia & - & - & - & - & - & - & - & - \\
\hline $\begin{array}{l}\text { Trastornos por comportamiento } \\
\text { disruptivo }\end{array}$ & 13,6 & 4,1 & 8,6 & 3,2 & 3,0 & 1,8 & 2,3 & 1,6 \\
\hline Trastorno disocial & - & - & - & - & 1,5 & 1,2 & 1,2 & 1,0 \\
\hline Trastornos oposicionista desafiante & 5,8 & 2,3 & 1,7 & 0,8 & 1,9 & 1,3 & 1,2 & 1,0 \\
\hline TDAH & 8,7 & 2,4 & 7,4 & 3,1 & 0,8 & 0,7 & 0,8 & 0,7 \\
\hline $\begin{array}{l}\text { Trastornos por consumo de alcohol } \\
\text { y sustancias }\end{array}$ & - & - & - & - & 7,7 & 2,8 & 2,5 & 1,5 \\
\hline Abuso de alcohol & - & - & - & - & 3,8 & 2,2 & 0,7 & 0,6 \\
\hline Dependencia de alcohol & - & -- & - & - & 2,1 & 1,5 & 1,8 & 1,4 \\
\hline Abuso de THC & - & - & - & - & 0,4 & 0,4 & - & - \\
\hline Dependencia de THC & - & - & -- & - & 0,5 & 0,5 & 0,5 & 0,5 \\
\hline Dependencia de nicotina & - & - & - & - & 2,6 & 2,1 & 0,5 & 0,5 \\
\hline Abuso de otras sustancias & - & - & - & - & 0,5 & 0,5 & - & - \\
\hline Dependencia de otras sustancias & - & - & - & - & 0,5 & 0,5 & - & - \\
\hline Esquizofrenia & 1,7 & 1,5 & - & - & - & - & - & - \\
\hline Cualquier diagnóstico & 27,6 & 4,1 & 16,7 & 2,8 & 28,7 & 6,2 & 16,8 & 5,4 \\
\hline
\end{tabular}


Epidemiología de trastornos mentales infanto-juveniles en la Provincia de Cautín - B. Vicente et al

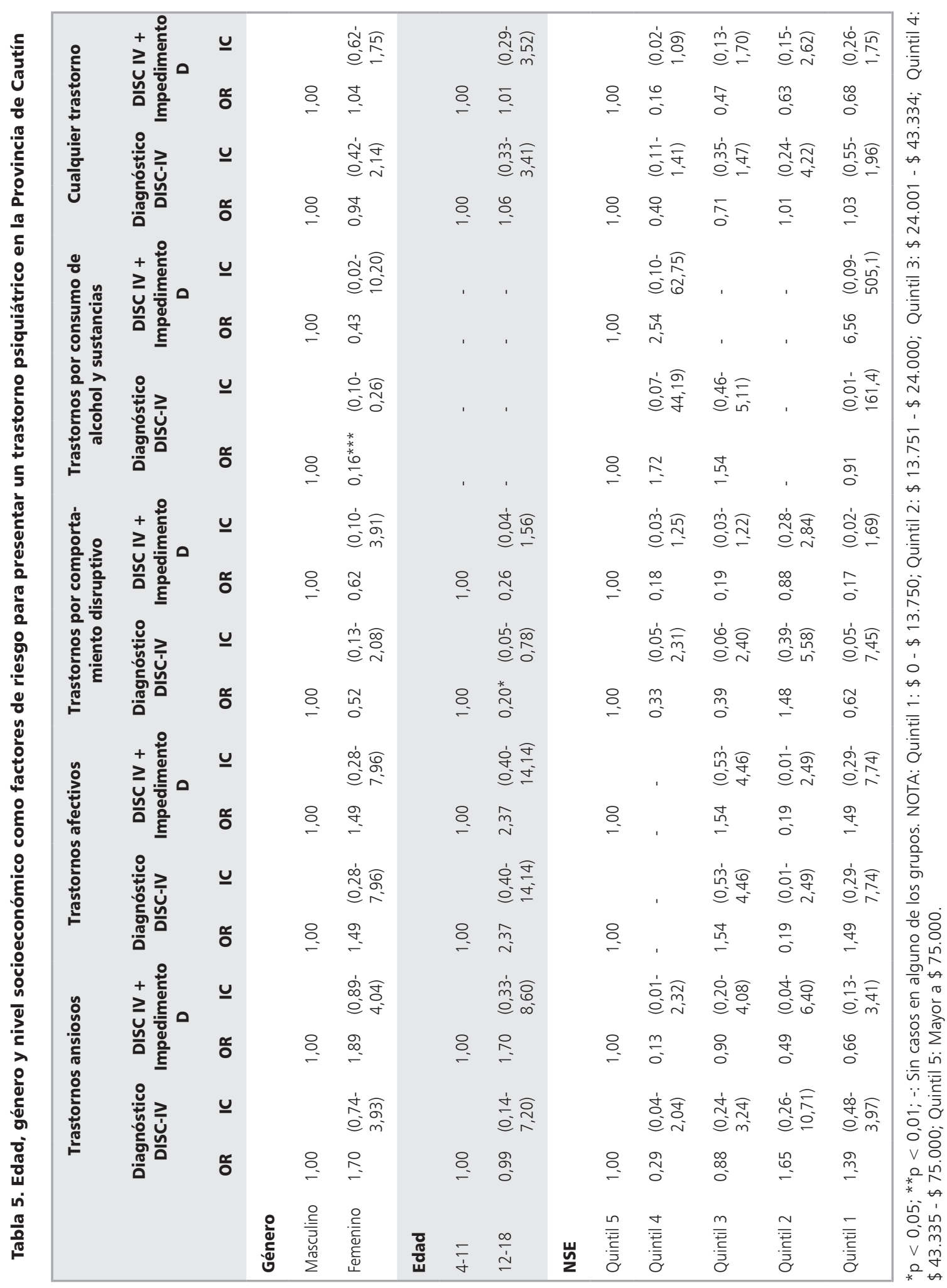


prevalencia de $7,7 \%$, que baja a 2,5\% al aplicar el criterio de discapacidad. No se encontró ningún trastorno de alimentación.

Al estudiar la asociación entre algunas variables demográficas y la presencia de patologías, sólo se encontró asociación entre pertenecer al género femenino y una menor probabilidad de presentar trastornos por consumo de alcohol y sustancias $(\mathrm{OR}=0,16 ; \mathrm{IC}=(0,10-0,26) ; \mathrm{p}<0,01)$; y entre ser adolescente y una menor probabilidad de presentar trastornos por comportamiento disruptivo $(\mathrm{OR}=0,20 ; \mathrm{IC}=(0,05-0,78) ; \mathrm{p}<0,05)$, como se observa en la Tabla 5.

\section{Discusión}

El artículo recoge los resultados de una de las provincias incluidas en el estudio nacional, en consecuencia, sus resultados no son extrapolables a la realidad nacional. Además, dado que no existen estudios poblacionales nacionales previos, toda comparación con los resultados publicados en la literatura nacional e internacional debe ser realizada con cautela.

La prevalencia global anual de trastornos psiquiátricos en la población estudiada alcanza a $28,1 \%$ previa a la aplicación de criterio de discapacidad. Ésta resulta mayor que la publicada por Canino y cols ${ }^{8}$ quienes, en un estudio utilizando el mismo instrumento en Puerto Rico, evaluaron a 1.886 menores entre 4 y 17 años obteniendo una prevalencia bruta de 19,8\%; y también mayor que la informada por Anderson y cols en Nueva Zelandia ${ }^{4}$ quienes utilizando el DISC-C, que arroja diagnóstico DSM-III, encontraron una prevalencia de $17,6 \%$.

Si se comparan los resultados con los publicados en los escasos estudios nacionales que entregan diagnóstico, con la cautela ya explicitada, la prevalencia global de $28,1 \%$ se aproxima a la publicada por Toledo y cols, para los alumnos de $1^{\circ}$ básico $(24,2 \%)^{9}$, y a la publicada por de la Barra y cols, para los alumnos de $1^{\circ}$ básico que mostraba $27,2 \%$. Pero es notablemente menor que la informada por la misma autora para $6^{\circ}$ básico donde la prevalencia llegaba a $45,7 \%{ }^{12}$. En ambos casos son muestras de extraídas de población escolar de un área delimitada de la ciudad de Santiago y los diagnósticos se basan en entrevistas clínicas sistematizadas efectuadas por médicos especialistas.

Otro aspecto a considerar es la disminución de la prevalencia al utilizar los criterios de impedimento. La prevalencia global disminuye desde $28,1 \%$ a $16,8 \%$ al aplicar el criterio $\mathrm{D}$ de discapacidad, el más estricto. Este descenso es mayor que el publicado por Canino y cols, quienes demuestran una disminución en la prevalencia de $19,8 \%$ a $16,4 \%$ al utilizar los criterios de discapacidad del mismo instrumento ${ }^{8}$, pero menor a lo encontrado por de la Barra y cols, que describen un descenso en la prevalencia desde un $45,7 \%$ a $15,7 \%$, al utilizar criterios para discapacidad ${ }^{12}$. La incorporación de criterios de discapacidad, permite seleccionar a partir del grupo de menores con sintomatología suficiente para diagnosticar una patología a aquellos con un nivel de compromiso (sufrimiento o discapacidad) clínicamente significativo, que hace necesaria, casi de regla, una intervención especializada.

Al analizar patologías específicas, el grupo de diagnósticos más prevalentes fue el de los trastornos ansiosos, con 18,9\% corregido a $8,7 \%$ con criterio de discapacidad. Los trastornos por comportamiento disruptivo son la segunda patología más frecuente, con cifras de 8,6\% que disminuye a $5,1 \%$ al corregir por discapacidad. El resultado es inverso al encontrado por Canino y $\operatorname{cols}^{8}$, quienes reportan una prevalencia más alta de trastornos disruptivos, seguido por los trastornos ansiosos.

$\mathrm{Si}$ se comparan las prevalencias por género se observan claras diferencias. Si bien en ambos grupos los trastornos ansiosos son los más frecuentes, la prevalencia en mujeres alcanza casi al doble que en los varones $y$, al corregir por discapacidad, en estos últimos los trastornos por comportamientos disruptivos, y el déficit atencional específicamente, aparecen como más frecuentes, mientras en las mujeres adquiere importancia la depresión mayor como patología específica. Esto concuerda con la literatura, en el sentido de identificar un perfil epidemiológico distinto donde predominan los cuadros disruptivos del comportamiento en los varones, y los cuadros afectivos en las mujeres ${ }^{29}$.

El análisis por grupos etarios muestra una mayor prevalencia de cuadros por comportamiento disruptivo, y TDAH en particular, en el grupo de menor edad y un aumento de patología depresiva en el grupo de adolescentes, junto a la ausencia de trastornos por uso de alcohol y sustancias en los más pequeños. Los hallazgos, a excepción de los relacionados con alcohol y sustancias que no fueron estudiados en dicha muestra, son consistentes 
con lo reportado por De La Barra y cols ${ }^{12}$ entre los alumnos de $1^{\circ}$ y $6^{\circ}$ básico. Sin embargo, es importante consignar que en nuestro estudio la fuente de información fue distinta en los dos grupos etarios, por lo que alguna proporción de las diferencias podrían atribuirse, al menos parcialmente, a la dificultad en los adolescentes para percibir problemas conductuales en ellos mismos, así como a la dificultad de los padres y/o cuidadores para identificar síntomas depresivos y/o de consumo de alcohol y drogas en sus hijos pequeños. Este aspecto constituye una de las limitaciones de nuestro estudio que puede ser salvada al incorporar al análisis los resultados de cuestionarios aplicados a los padres de los adolescentes.

Desde un punto de vista más global, llama la atención que la disminución de las prevalencias al utilizar criterios de discapacidad es notoria en los trastornos ansiosos, mucho menor en los trastornos disruptivos, e inexistente en los trastornos afectivos. Es probable que cuando algunas patologías, como los trastornos del ánimo, alcanzan criterios clínicos suficientes para ser diagnosticadas, impliquen también una gravedad suficiente que genera discapacidad. En cambio, muchos de los trastornos ansiosos que cumplen criterios diagnósticos pueden no tener la gravedad suficiente para asociarse a discapacidad. Ello abre la discusión acerca de la pertinencia de tratar o no a estos menores, cuya sintomatología es por el momento no invalidante, y su impacto futuro debe ser estudiado en profundidad.

Finalmente, las dos únicas asociaciones encontradas, la menor probabilidad de presentar trastornos del comportamiento en el grupo de mayor edad y trastornos por abuso de sustancias en el sexo femenino, pierden validez estadística al incorporar criterio de discapacidad. Ello no confirma los hallazgos de Canino y cols ${ }^{8}$, quienes encontraron una asociación positiva entre sexo masculino y trastornos del comportamiento, particularmente déficit atencional; y una asociación negativa entre sexo masculino y el grupo de los trastornos afectivos, y depresión mayor en particular; así como una relación positiva entre edad y trastornos ansiosos y del ánimo; relaciones demostradas una vez aplicado el criterio de discapacidad.

Pese a las limitaciones inherentes al estudio, como el tamaño muestral, que dificulta la obtención de diferencias estadísticamente significativas, y el hecho que sólo se utilizó el auto-reporte en los adolescentes, la presente investigación da cuenta de una muestra rigurosamente diseñada que es representativa de la totalidad de la población entre 4 y 18 años de la provincia de Cautín, constituyendo de esta manera los primeros resultados de prevalencia de patologías psiquiátricas en población general infanto-juvenil publicados para el país. La importancia de estos hallazgos radica en que permiten, desde ya, contar con una base objetiva para la planificación y asignación de recursos en Salud Mental del niño y del adolescente, permitiendo tanto la formación y capacitación de recursos humanos en áreas prioritarias como la asignación de recursos a grupos específicos con mayores vulnerabilidades para ciertas patologías. Por otra parte, aunque estos hallazgos son representativos sólo de una provincia del país, constituyen la primera aproximación al conocimiento de la real magnitud del problema de salud mental en la población infantojuvenil chilena.

\section{Referencias}

1. Araya R, Rojas G, Fritsch R, Acuña, Lewis G. Common mental disorders in Santiago, Chile. Br J Psychiat 2001; 178: 228-33.

2. Vicente B, Rioseco P, Saldivia S, Kohn R, Torres S. Estudio chileno de epidemiología psiquiátrica (ECEP). Rev Med Chile 2002; 130: 527-36.

3. Vicente B, Kohn R, Rioseco P, Saldivia S, Baker CL, Torres S. Psychiatric Prevalence Study. Population prevalence of psychiatric disorders in Chile: 6-month and 1-month rates. Br J Psychiat 2004; 184: 299-305.

4. Anderson JC, William S, Mc Gee R, Silva PA. DSM-III disorders in preadolescent children. Arch Gen Psychiat 1987; 44: 69-76.

5. Bird H, Canino G, Rubio-Stipec M, Gould M, Ribera J, Sesman M, et al. Estimates of the prevalence of childhood maladjustment in a community survey in Puerto Rico: the use of combined measures. Arch Gen Psychiat 1988; 45: 1120-6.

6. Mc Gee R, Fecham M, William S, Fortridge F, Silva P, Kelly J. DSM-III disorder in a large sample of adolescents. J Am Acad Child Adolesc Psychiat 1990; 29: 611-9.

7. Puura K, Almqvist F, Tamminent T, Piha J, Rasamen E, Kumpulainen K, et al. Psychiatric distrubances among prepuberal children in South Finland. Soc. Psychiatry Psychiatry Epidem 1998; 33: 310-8.

8. Canino G, Shrout PE, Rubio-Stipec M, Bird HR, Bravo M, Ramírez R, et al. The DSM-IV Rates of Child and 
Adolescent Disorders in Puerto Rico. Prevalence, Correlates, Service Use, and the Effects of Impairment. Arch Gen Psychiatry 2004; 61: 85-93.

9. Toledo V, De La Barra F, López C, George M, Rodríguez J. Diagnóstico psiquiátrico de una cohorte de escolares de primer año de enseñanza básica del Área Occidente de Santiago. Rev Chil Neuro-Psiquiat 1997; 35: 17-24.

10. Bralic S, Seguel X, Montenegro H. Prevalencia de trastornos psíquicos en la población escolar de Santiago de Chile. Acta Psiquiátrica y Psicológica de América Latina 1987; 33: 316-25.

11. De La Barra F, Toledo V, Rodríguez J. Estudio de salud mental en dos cohortes de niños escolares de Santiago Occidente. I: Prevalencia y seguimiento de problemas conductuales y cognitivos. Rev Chil Neuro-Psiquiatr 2002; 40: 9-21.

12. De La Barra F, Toledo V, Rodríguez J. Estudio de salud mental en dos cohortes de niños escolares de Santiago Occidente. IV: desórdenes psiquiátricos, diagnóstico psicosocial y discapacidad. Rev Chil Neuro-Psiquiat 2004; 42: 259-72.

13. Almonte C, Capurro G. Estructura de la Morbilidad Psiquiátrica en niños y adolescentes en consulta ambulatoria y Hospitalización. Psiquiatría y Salud Mental 2001; 18: 22-8.

14. Recart C, Castro P, Álvarez H, Bedregal P. Características de niños y adolescentes atendidos en un consultorio psiquiátrico del sistema privado de Salud en Chile. Rev Med Chile 2002; 130: 295-303.

15. Kish L. Survey Sampling. New York, NY, John Wiley \& Sons; 1965.

16. Costello AJ, Edelbrock CS, Duncan MK, Kalas R (1984). Las pruebas del NIMH Entrevista Diagnóstica para Niños (DISC) en una población clínica. Rockville, MD: Centro de Estudios Epidemiológicos, Informe del Instituto Nacional de Salud Mental.

17. Herzog, David B. Sensitivity of the Diagnostic Interview Schedule for Children, 2nd edition (DISC-2.1) for specific diagnoses of children and adolescents. Journal of the American Academy of Child and Adolescent Psychiatry 1993; 32: 666-73.

18. Schwab-Stone M, Fallon T, Briggs M, Crowther B. Reliability of diagnostic reporting for children aged 6-11 years: a test-retest study of the Diagnostic Interview Schedule for Children-Revised. Am J Psychiatry 1994; 151: 1048-54.

19. Schwab-Stone M, Shaffer D. Criterion Validity of the NIMH Diagnostic Interview Schedule for Children Version 2.3 (DISC-2.3). J Am Acad Child Adolescent Psychiatry 1996; 35: 878-88.
20. Shaffer D, Fisher P, Dulcan MK, Davies M, Piacentini J, Schwab-Stone ME, et al. The NIMH Diagnostic Interview Schedule for Children Version 2.3 (DISC-2.3): description, acceptability, prevalence rates, and performance in the MECA Study: Methods for the Epidemiology of Child and Adolescent Mental Disorders Study. J Am Acad Child Adolesc Psychiatry 1996; 35: 865-77.

21. Shaffer D, Fisher P, Dulcan MK, et al. The NIMA Diagnostic Interview Schedule for Children Version 2.3(DISC-2.3): description, acceptability, prevalence rates, and performance in the MECA study. J Am Acad Child Adolesc Psychiat 1996; 35: 865-77.

22. Shaffer D, Schwab-Stone M, Fisher P, Cohen P, Piacentini J, Davies $\mathrm{M}$, et al. The Diagnostic Interview Schedule for Children-Revised Version (DISC-R): I. Preparation, Field Testing, Interrater Reliability, and Acceptability. J Am Acad Child Adolesc Psychiat 1993; 32: 3.

23. Shaffer D, Fisher P, Lucas C, Dulcan M, Schwab-Stone M. NIMH Diagnostic Interview Schedule for Children Version IV (NIMH DISC-IV): Description, Differences From Previous Versions, and Reliability of Some Common Diagnoses. J Am Acad Child Adolesc Psychiat 2000; 39: 28-38.

24. Shaffer D, Fisher P, Lucas CP, Dulcan Mk, Schwab-Stone ME. NIMH Diagnostic Interview Schedule for Children VersionIV (NIMH DISC-IV): description, differences from previous versions, and reliability of some common diagnoses. J Am Acad Child Adolesc Psychiat 2000; 39: 28-38.

25. Hough RL. Comparing DISC-IV and clinician Diagnoses among youths receiving public mental health services. Journal of the American Academy of Child and Adolescent Psychiatry. J Am Acad Child Adolesc Psychiat 2003; 42: 349-56.

26. Karno M, Burman A, Escobar J, Hough R, Eaton W. Development of the Spanish-Language Version of the National Institute of Mental Health Diagnostic Interview Schedule. Arch Gen Psychiatry 1983; 40: 1183-8.

27. Canino G, Bird H. The Spanish Diagnostic Interview Schedule: Reliability and Concordance with Clinical Diagnosis in Puerto Rico. Arch Gen Psychiat 1987; 44: 720-726.

28. Bravo M, Ribera J, Rubio-Stipec M, Canino G, Shrout P, Ramírez R, et al. Test-retest reliability of the Spanish version of the Diagnostic Interview Schedule for Children (DISC-IV). J Abnorm Child Psychol 2001; 29: 433-44.

29. Polanczyk G, Silva de Lima M, Horta B, Biederman J, Rohde L. The worldwide prevalence of ADHD: A systematic review and metaregression analysis. Am J Psychiatry 2007; 164: 942-8. 